\section{A genome-engineered bioartificial implant for autoregulated anticytokine drug delivery}

\author{
Yun-Rak Choi ${ }^{1,2,3,4 \dagger}$, Kelsey H. Collins ${ }^{1,2,3 \dagger}$, Luke E. Springer ${ }^{5}$, Lara Pferdehirt ${ }^{1,2,3,6}$, \\ Alison K. Ross ${ }^{1,2,3,6}$, Chia-Lung Wu ${ }^{1,2,3 \neq}$, Franklin T. Moutos ${ }^{7}$, Natalia S. Harasymowicz ${ }^{1,2,3}$, \\ Jonathan M. Brunger ${ }^{8,9}$, Christine T. N. Pham ${ }^{5}$, Farshid Guilak ${ }^{1,2,3,6,7 *}$
}

\begin{abstract}
Biologic drug therapies are increasingly used for inflammatory diseases such as rheumatoid arthritis but may cause significant adverse effects when delivered continuously at high doses. We used CRISPR-Cas9 genome editing of iPSCs to create a synthetic gene circuit that senses changing levels of endogenous inflammatory cytokines to trigger a proportional therapeutic response. Cells were engineered into cartilaginous constructs that showed rapid activation and recovery in response to inflammation in vitro or in vivo. In the murine $\mathrm{K} / \mathrm{BxN}$ model of inflammatory arthritis, bioengineered implants significantly mitigated disease severity as measured by joint pain, structural damage, and systemic and local inflammation. Therapeutic implants completely prevented increased pain sensitivity and bone erosions, a feat not achievable by current clinically available disease-modifying drugs. Combination tissue engineering and synthetic biology promises a range of potential applications for treating chronic diseases via custom-designed cells that express therapeutic transgenes in response to dynamically changing biological signals.
\end{abstract}

\section{INTRODUCTION}

Disease-modifying antirheumatic biologic drugs have revolutionized the treatment of autoimmune diseases; however, approximately $40 \%$ of patients with rheumatoid arthritis (RA) fail to respond to treatment (1). Moreover, antirheumatic biologic drugs suppress the immune system, predisposing patients to substantial adverse effects (AEs), such as increased risk of infection (2). Biologic drugs are generally designed to target several inflammatory cytokines and pathways, including interleukin-1 (IL-1), IL-6, and tumor necrosis factor- $\alpha$ (TNF- $\alpha$ ). While the severity of RA fluctuates over time, these drugs are administered continuously at high concentrations, regardless of endogenous cytokine levels or the kinetics of RA symptoms. The development of specific therapeutic strategies that can sense and respond to endogenous levels of inflammatory mediators could overcome some of the challenges observed with constant delivery of cytokine inhibitors and mitigate unwanted AEs (3-6).

Here, we used CRISPR-Cas9 genome engineering $(7,8)$ to create such a self-regulating synthetic gene circuit in induced pluripotent stem cells (iPSCs). These cells were engineered into a bioartificial implant and evaluated in vitro and in vivo for their ability to produce anticytokine biologic drugs in response to inflammatory signals such as IL-1 or TNF- $\alpha$ by transcribing their biologic inhibitors in a feedbackcontrolled manner (3), driven by the promoter of chemokine (C-C) motif ligand 2 (Ccl2) (9). For proof of concept as a disease therapy,

\footnotetext{
Department of Orthopaedic Surgery, Washington University in St. Louis, St. Louis MO 63110, USA. ${ }^{2}$ Shriners Hospitals for Children, St. Louis, MO 63110, USA. ${ }^{3}$ Center of Regenerative Medicine, Washington University in St. Louis, St. Louis, MO 63110 USA. ${ }^{4}$ Department of Orthopaedic Surgery, Yonsei University College of Medicine, Seoul, South Korea. ${ }^{5}$ Division of Rheumatology, Department of Medicine, Washington University in St. Louis, St. Louis, MO 63110, USA. ${ }^{6}$ Department of Biomedical Engineering, Washington University in St. Louis, St. Louis, MO 63130, USA. ${ }^{7}$ Cytex Therapeutics Inc., Durham, NC 27704, USA. ${ }^{8}$ Department of Biomedical Engineering, Vanderbilt University, Nashville, TN 37235, USA. ${ }^{9}$ Center for Stem Cell Biology, Vanderbilt University, Nashville, TN 37235, USA.

*Corresponding author. Email: guilak@wustl.edu

tThese authors contributed equally to this work.

$\ddagger$ Present address: Department of Orthopaedic Surgery and Rehabilitation, Center for Musculoskeletal Research, University of Rochester, Rochester, NY, USA.
}

we delivered the bioengineered implants in the murine model of $\mathrm{K} / \mathrm{BxN}$ inflammatory arthritis, which has been shown to be critically dependent on IL-1 (10). Although IL-1 receptor antagonist (IL-1Ra) has been shown to mitigate disease in animal models of RA and slows joint damage (assessed radiographically) in humans (11), it is not routinely used as a disease-modifying biologic in RA because of its short half-life and modest effects (12). We posit that delivering IL-1Ra using a system that can sense and respond to dynamically changing levels of endogenous inflammatory mediators may improve efficacy while mitigating the side effects of continuous biologic delivery.

Our results show that dynamic, autoregulated delivery of IL-1Ra mitigated inflammation, pain, and structural damage in $\mathrm{K} / \mathrm{BxN}$ arthritis that was superior to conventional disease-modifying antirheumatic drugs (DMARDs) in this context. This therapeutic approach completely abrogated bone erosions as compared to conventional disease-modifying drugs and biologics, which is yet to be achieved by daily subcutaneous injection of IL-1Ra (anakinra). Furthermore, these therapeutic constructs could be reactivated repeatedly, following in vivo inflammatory triggers (such as RA flares), a clinically important facet of this approach that has relevant translational impact for long-term drug delivery using this approach.

\section{RESULTS}

\section{Cell-based injection of genome-engineered iPSCs sense} and respond to inflammation in vivo

Using CRISPR-Cas9 genome engineering, iPSCs were edited to insert either the gene for IL-1Ra $(\mathrm{Ill} / \mathrm{rn})$ or luciferase $(\mathrm{Luc})$ at the $\mathrm{Ccl} 2$ locus, creating a self-regulating gene circuit that transcribes $\mathrm{Il} 1 \mathrm{rn}$ or $\mathrm{Luc}$ in response to inflammatory activation of $\mathrm{Ccl} 2$ (referred as Ccl2-IL1Ra or Ccl2-Luc cells; Fig. 1) (3). We initially examined the therapeutic potential of Ccl2-Luc cells (13) by intraperitoneal injection into the $\mathrm{K} / \mathrm{BxN}$ murine model of arthritis (10). This model has a well-characterized IL-1-dependent development of spontaneous arthritis in the F1 generation that is observed starting from 3 to 4 weeks and progressing to advanced disease at 7 to 8 weeks of age (14). When Ccl2-Luc cells were delivered via intraperitoneal injection to 


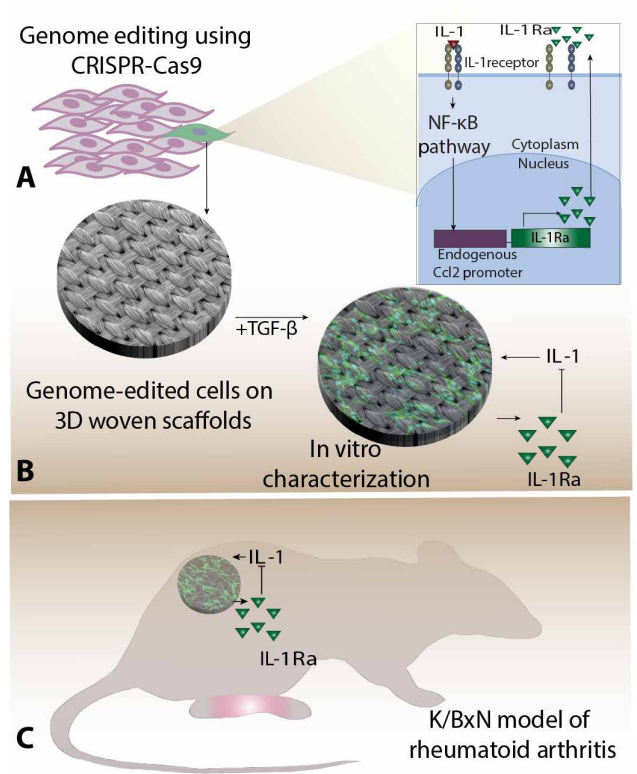

Fig. 1. Scaffold-mediated delivery of genome-engineered anticytokine cells. (A) CRISPR-Cas9 genome editing was used to engineer stem cells containing a synthetic gene circuit that expresses the IL-1 Ra, an inhibitor of IL-1, in response to activation of the chemokine (C-C) motif ligand 2 ( $\mathrm{C} / 2)$ promoter. NF- $\mathrm{kB}$, nuclear factor $\mathrm{\kappa B}$. (B) Cells were engineered to form stable cartilaginous constructs on three-dimensional (3D) woven scaffolds in chondrogenic media. Constructs were tested in vitro to assess on-off dynamics in response to changing levels of IL-1. (C) To test their efficacy, these anticytokine constructs were implanted subcutaneously in $\mathrm{K} / \mathrm{BxN}$ model of RA.

mice 4 and 8 weeks of age, we detected luciferase signal at 1 hour after implantation that persisted over 24 hours of monitoring (Fig. 2A). Older K/BxN F1 mice demonstrated increased luminescence intensity compared to the younger mice, concordant with the increased clinical score observed in 8-week-old versus 4-week-old mice (Fig. 2, B and C). Luminescence intensity observed at 24 hours after injection demonstrated a strong positive relationship with clinical scores across all animals (Spearman's $r=0.93, P=0.033$ ), indicating that these cells can sense and proportionately respond to their environment and can provide a real-time luminescence readout of systemic inflammation.

Next, Ccl2-Luc (controls) and Ccl2-IL1Ra cells were delivered intraperitoneally and tested in the context of $\mathrm{K} / \mathrm{BxN}$ serum transfer arthritis (STA) (15), a transient arthritis that persists for 7 to 14 days in wild-type mice. Intraperitoneally injected bioengineered cells were no longer detectable 24 hours after injection. While the control Ccl2-Luc cells did not have an effect on K/BxN STA (Fig. 2D), Ccl2IL1Ra cell delivery resulted in small but significant and sustained improvement in ankle thickness, tactile allodynia, and pressure-pain hyperalgesia at the ankle, despite being cleared 24 hours after injection. Intraperitoneally injected Ccl2-IL1Ra cells, however, did not mitigate clinical scores $(P=0.10$; Fig. $2 \mathrm{E})$.

\section{Tissue-engineered bioartificial implants sense and respond to inflammation in a feedback-controlled manner}

To create a stable cell-based implant, Ccl2-IL-1Ra and Ccl2-Luc cells were seeded onto three-dimensional (3D) woven poly( $\varepsilon$-caprolactone) (PCL) scaffolds (Fig. 3A) (16) and chondrogenically differentiated (17) over 21 days into chondrocyte-like cells that produced a proteoglycan-rich matrix (Fig. 3, B to D). This approach was used because chondrocyte-like cells within a cartilage matrix exhibit minimal migration and require neither vasculature nor innervation for long-term survival, relying on diffusion for nutrient transport even under physiologically relevant hypoxic conditions (18-21). In culture, the Ccl2-IL1 Ra bioartificial implants rapidly sensed and responded to IL- $1 \alpha$ stimulation by producing IL-1Ra (Fig. 3E) in a feedback-controlled manner (Fig. 3, F and G). A second set of cells were similarly engineered to produce soluble TNF receptor 1 (sTNFR1), and implants created from Ccl2-sTNFR1 cells behaved similarly in vitro (fig. S1). The cell-secreted anticytokine biologics suppressed the response to IL- $1 \alpha$ or TNF- $\alpha$ stimulation, reducing mRNA levels for proinflammatory mediators $\mathrm{Il} 6$ and $\mathrm{Ccl} 2$, and for matrix metalloproteinases, $M m p 9$ and $M m p 13$ (Fig. 3, $\mathrm{H}$ to K, and fig. S1), as compared to the control Ccl2-Luc construct. Bioartificial implants demonstrate stability as a long-term drug
delivery system and demonstrate retriggering dynamics in vivo These bioartificial constructs were implanted subcutaneously in $\mathrm{K} / \mathrm{BxN}$ transgenic F1 mice, which develop spontaneous arthritis, and mice with $\mathrm{K} / \mathrm{BxN}$ STA (15). Ccl2-Luc constructs exhibited high luminescence relative to background levels, indicating responsiveness of the constructs in vivo, and cell survival in a chronic inflammatory environment up to 5 weeks after implantation in $\mathrm{F} 1 \mathrm{~K} / \mathrm{BxN}$ mice (Fig. 4, A and B). In the $\mathrm{K} / \mathrm{BxN}$ STA model, Ccl2-Luc constructs exhibited similar luminescence signals for at least 7 days when they were implanted subcutaneously near the knee or on the dorsum of mice (Fig. 4C). Explanted Ccl2-Luc constructs from F1 K/BxN mice at 5 weeks also confirmed high cell viability ( $70 \%$ on average) for both Ccl2-IL1Ra and Ccl2-sTNFR1 constructs, via live/dead staining by confocal microscopy (Fig. 4D).

We then determined the activation and reactivation dynamics of this genetically engineered drug delivery platform. First, we implanted Ccl2-Luc constructs into mice and challenged them 7 days later with $\mathrm{K} / \mathrm{BxN}$ serum administration to induce STA (Fig. 4E). Significant increases in luminescence were detected as early as 1 hour after challenge with $\mathrm{K} / \mathrm{BxN}$ serum in vivo (Fig. $4 \mathrm{~F}$ ). We observed that the photon flux measured by IVIS imaging was concordant with the clinical score, such that when the inflammatory arthritis flare waned at days 9 to 14 , the luminescence intensity also decreased, demonstrating in vivo on-off kinetics proportional to endogenous inflammatory signals (Fig. 4G). Furthermore, these constructs were able to be reactivated after a week-long washout period to similar photon flux levels observed in response to the first $\mathrm{K} / \mathrm{BxN}$ serum transfer. Ccl2-IL1Ra therapeutic implants maintained their efficacy after 30 days of dormancy, exhibiting significant mitigation of clinical disease severity when rechallenged with $\mathrm{K} / \mathrm{BxN}$ serum (Fig. $4, \mathrm{H}$ and I). After implantation, Ccl2-Luc constructs maintained a fibrocartilaginous phenotype, illustrated by low Safranin O staining and robust Col II labeling using immunohistochemistry, and positive staining for Col I and Col X (Fig. 4J). The constructs exhibited increased cellularity [hematoxylin and eosin (H\&E) staining] but did not show any vascularity, immune cell invasion, or calcification.

\section{Bioartificial implants mitigate disease severity and pain in mice with inflammatory arthritis}

To determine the ability of this self-regulating implant to mitigate arthritis in the STA model, constructs were implanted on the dorsal aspect of C57BL/6 mice (day -7) and allowed to recover before 
A

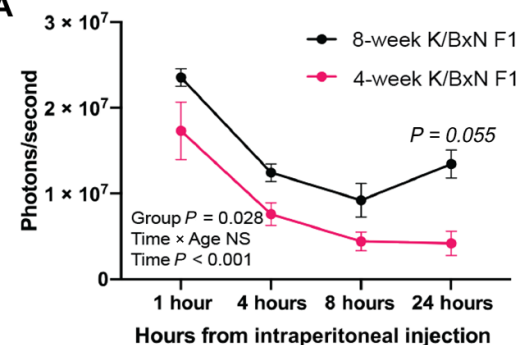

B

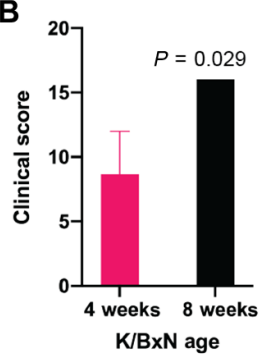

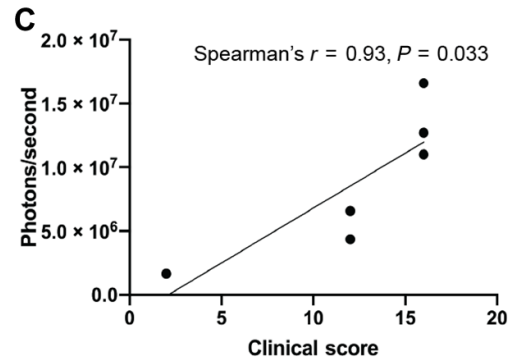

D

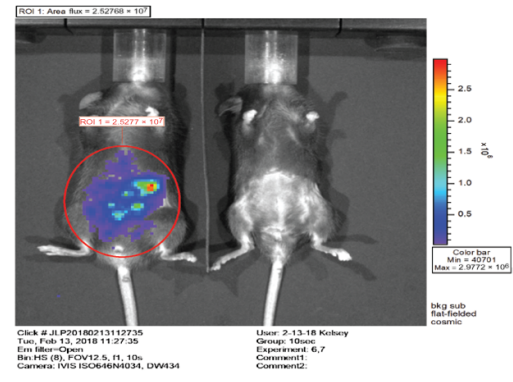

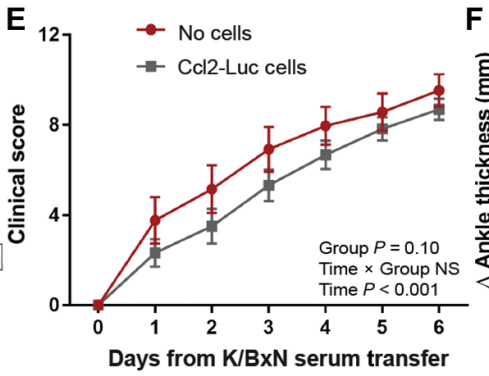

$\mathbf{F}$

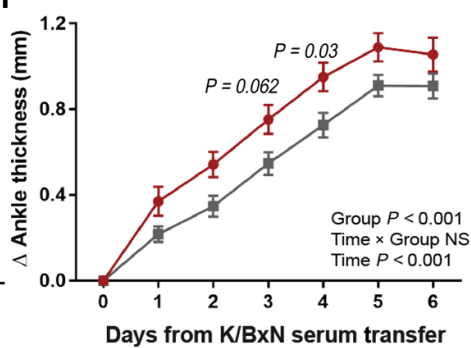

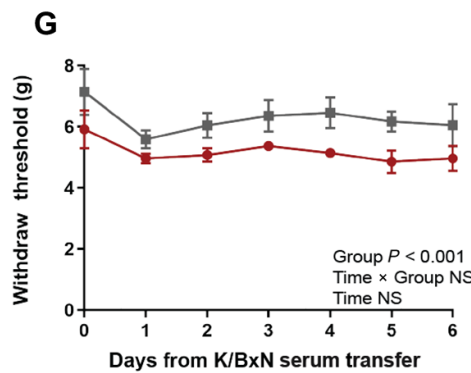

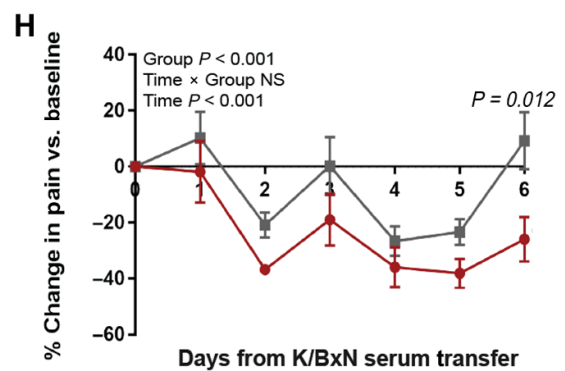

Fig. 2. Genome-edited cells were tested for therapeutic efficacy using an intraperitoneal injection. (A) Eight-week-old K/BxN F1 mice demonstrated increased luciferase signal over 24 hours, and (B) demonstrated increased disease burden compared to 4-week-old mice, ( $n=3$ to 4$)$, (C) which had a significant positive association with luminescence intensity. (D) Rapid luciferase expression (within 1 hour) in response to supraphysiological levels of TNF- $\alpha$ (left; right, no TNF- $\alpha$ challenge). (E) Significant mitigation of clinical scores was not observed $(P=0.10)$ with K/BXN STA after intraperitoneal injection of Ccl2-IL1Ra cells $(\mathbf{F})$ but did significantly mitigate ankle thickness. Intraperitoneally injected CCl2-IL1Ra cells also mitigated pain measured by (G) electronic Von Frey and (H) algometry in K/BxN STA mice. Values represent means \pm SEM. Analysis: (A and E to H) Two-way repeated measures analysis of variance (ANOVA), after confirming normality, with a Geisser-Greenhouse correction. Exact $P$ values are demonstrated for the main effect of group, except for when $P<0.001$, as indicated. Interaction terms and time are demonstrated in the graphs. Not significant (NS), $P>0.05$. Significant Sidak's post hoc test per time point between groups with an italicized adjusted $P$ value directly over each time point is shown. (B) Mann-Whitney $U$ test after a significant $f$ test was identified. In (C), Spearman correlation was calculated between scores and luminescence intensity.

intravenous $\mathrm{K} / \mathrm{BxN}$ serum administration on day 0 (Fig. 5A). Animals receiving Ccl2-IL1Ra implants demonstrated significant amelioration of arthritis severity, including $40 \%$ reductions in both clinical scores and ankle thickness, compared to control animals that received Ccl2-Luc constructs or no implant (Fig. 5, B and C).

Histologic analysis illustrated that animals with Ccl2-IL1Ra implants demonstrated significantly lower inflammation scores and had reduced cartilage degradation and proteoglycan loss (Fig. 5, D to F) when compared to animals with Ccl2-Luc implants. Mice receiving Ccl2-IL1Ra constructs also exhibited significantly higher serum levels of IL-1Ra as compared to controls (Fig. 5, G to H). Serum IL-1Ra concentrations were correlated with clinical scores and ankle thickness (Fig. 5, I and J).

The reduction in disease severity in mice with Ccl2-IL1Ra constructs was also accompanied by a significant decrease in mechanical pain sensitivity as measured by algometry and electronic Von Frey pain tests, whereas the control groups exhibited increased pain sensitivity (reduced pain thresholds) (Fig. 5, K and L). The pain threshold of each animal was also significantly correlated with serum levels of IL-1Ra (Fig. 5, M and N). Ccl2-sTNFR1 (fig. S3) or reduced dose of Ccl2-IL1Ra constructs (four scaffolds versus eight scaffolds; fig. S3) did not significantly mitigate K/BxN STA clinical severity, although both approaches significantly attenuated the increase in pain sensitivity (figs. S2 and 3). There was also evidence of a dose-dependent response in the clinical score and circulating levels for IL-1Ra with implantation of half the dose of Ccl2-IL1Ra constructs (four scaffolds; fig. S3). These data corroborate previous reports that TNF- $\alpha$ and IL- $1 \alpha$ signaling differentially affect inflammation and pain in K/BxN STA (10).

\section{Bioartificial implants prevent bone and joint destruction in mice with $\mathrm{K} / \mathrm{BxN}$ STA}

Micro-computed tomography (microCT) of bone structure showed that mice receiving Ccl2-IL1Ra implants were completely protected from bone erosions that are characteristic of inflammatory arthritis. Treated mice had minimal or no bone erosions on day 7 following 
A

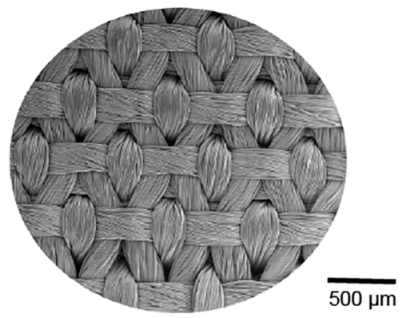

E
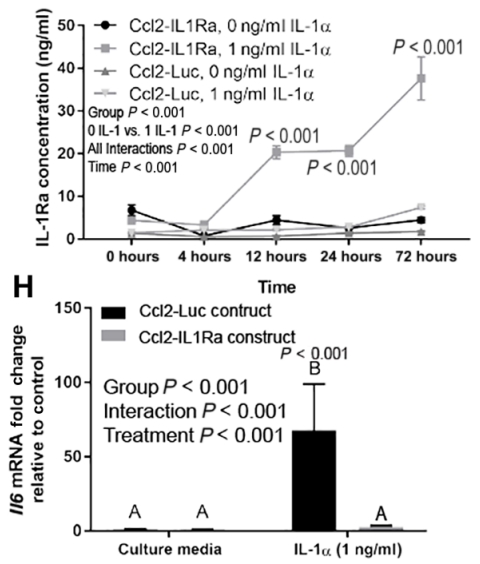

B

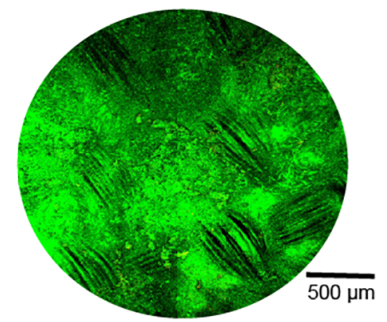

$\mathbf{F}_{\text {屁 }}$

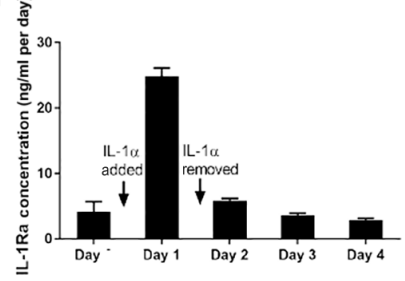

I

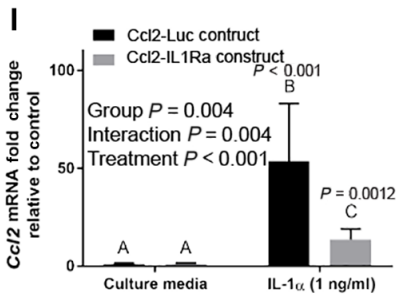

$\mathrm{C}$

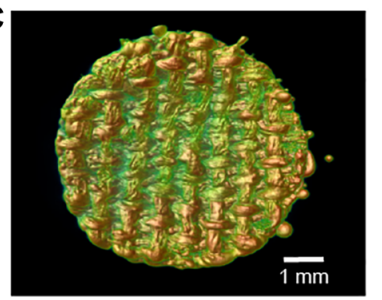

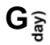

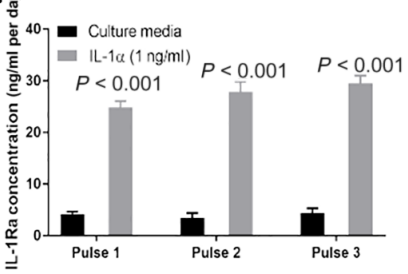

J

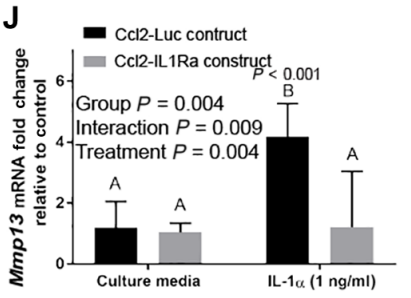

D

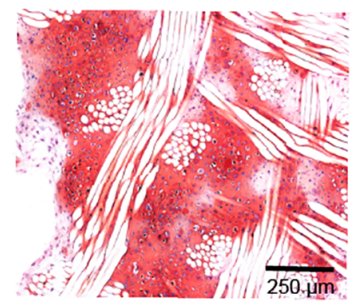

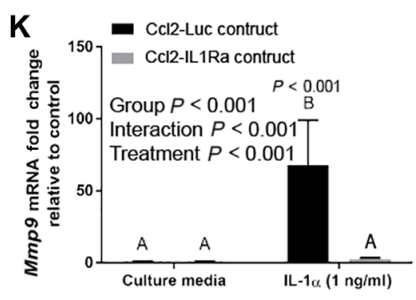

Fig. 3. Tissue-engineered bioartificial implants sense and respond to inflammation in a feedback-controlled manner. (A to $D)$ Genome-engineered stem cells on porous 3D woven scaffolds [(A) electron microscopy] were differentiated into chondrocyte-like cells that infiltrated the scaffold and filled the scaffold pores with cartilaginous matrix as shown by (B) confocal microscopy, (C) nano-computed tomography (nanoCT), (D) and Safranin O staining. (E) CCl2-IL1Ra constructs produced IL-1Ra in response to IL-1 $\alpha$ ( $n=5$ per time point) and (F) decreased IL-1 Ra production rapidly after IL- $1 \alpha$ withdrawal ( $n=5$ per day). (G) This response was repeatable over multiple cycles of iterative stimulation ( $n=5$ per group). ( $\mathbf{H}$ to $\mathbf{K})$ Ccl2-IL1Ra constructs $(n=6)$ mitigated mRNA levels for proinflammatory mediators as compared to Ccl2-Luc constructs $(n=5)$. Data in $(E)$ and $(G)$ were compared with two-way repeated measures ANOVA with Geisser-Greenhouse corrections and Sidak's post hoc test, where significant adjusted $P$ values per time point are illustrated in italics. Main effect $P$ values are displayed for each graph. Data in $(\mathrm{H})$ to $(\mathrm{K})$ were evaluated using a two-way ANOVA with Geisser-Greenhouse corrections and Sidak's post hoc test. Different letters indicate $P<0.05$, and exact $P$ values are shown.

K/BxN serum transfer, whereas mice with Ccl2-Luc implants or no implant exhibited highly erosive disease as demonstrated by a higher ratio of bone surface to bone volume $(\mathrm{BS} / \mathrm{BV})$ and a lower ratio of bone volume per total volume (BV/TV) (Fig. 6, A to C). Histologic analysis was also concordant with the microCT observations for bone erosions (Fig. 6D). Ccl2-IL1Ra implants also showed protective effects on bone mineral density (BMD) (Fig. 6D). Quantitative measures of bony erosions (BS/BV) were negatively correlated with serum levels of IL-1Ra (Fig. 6E).

Mice that were treated with Ccl2-IL1Ra implants showed significant reductions in their inflammatory cytokine profiles as compared to control animals. Using a multiplexed cytokine assay, we observed significant reductions of serum levels for interferon- $\gamma$ and IL- 6 and an increase in serum levels for IL-10 in treated animals (Fig. 6, F to H). In the paw lysate of treated animals, we observed significant reductions in levels for IL-1 $\alpha$, IL-6, Monocyte chemoattractant protein-1, keratinocytes-derived chemokine, also known as chemokine (C-X-C motif) ligand 1 (CXCL1), and TNF- $\alpha$ in comparison to control animals (Fig. 6, I to M). There was a trend toward reduction in total level of IL-1 (IL-1 $\alpha+$ IL-1 $\beta)$ in animals that received Ccl2-IL1Ra implants compared to controls $(P=0.07$; Fig. $6 \mathrm{~N})$.

\section{Bioartificial implants demonstrate improved clinical efficacy to standard-of-care treatment}

To benchmark this approach against several current DMARDs, we compared the clinical response of animals with $\mathrm{K} / \mathrm{BxN}$ STA receiving Ccl2-IL1Ra implants to those treated with methotrexate, a conventional DMARD, or tofacitinib, a Janus kinase (JAK) inhibitor (22). We saw no significant mitigation of arthritis with methotrexate or JAK inhibitor (Fig. 7, A and B), while Ccl2-IL1Ra constructs mitigated ankle thickness (Fig. 7, C and D) and prevented pressure-pain hyperalgesia (Fig. 7, E and F). Mice implanted with Ccl2-IL1Ra constructs maintained BV/TV (Fig. 7G) and had reduced bone erosions, operationalized by BS/BV (Fig. 7H), to levels of healthy paws. We also compared treatment with anakinra, a biologic drug inhibitor of IL-1, to cell-based delivery of IL-1Ra via Ccl2-IL1Ra implants (Fig. 7I). Despite exhibiting similar circulating levels of IL-1Ra, mice treated with a clinically relevant dose of anakinra did not show mitigation of arthritis, while mice that received Ccl2-IL1Ra implants demonstrated improved disease scores 6 days after $\mathrm{K} / \mathrm{BxN}$ serum transfer (Fig. 7J). These data suggest that dynamic cell-based release of therapeutic levels of IL-1Ra on demand in response to endogenous inflammatory signals results in improved cytokine inhibition as compared to twice daily injections of anakinra.

\section{DISCUSSION}

In this study, we combined the principles of synthetic biology and tissue engineering to develop a genome-engineered implantable drug delivery system that can automatically sense and respond to inflammatory cytokines to produce therapeutic levels of anticytokine biologic 
A

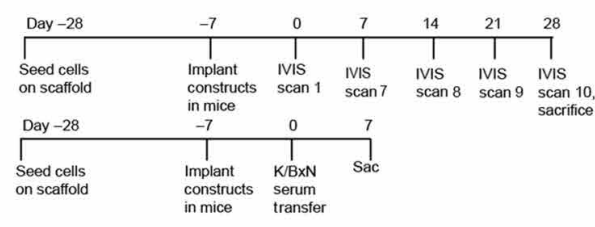

B

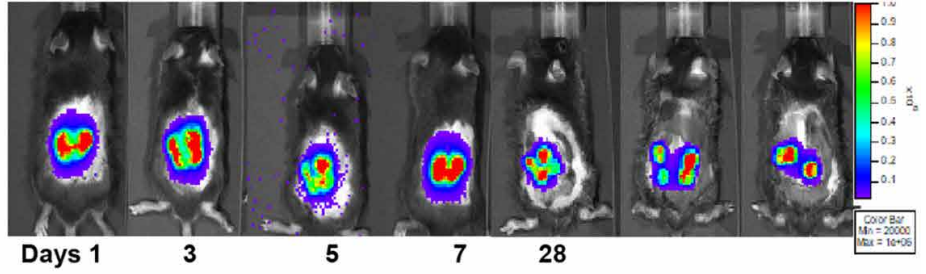

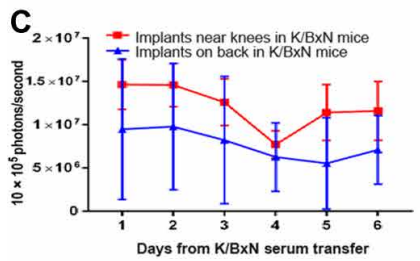
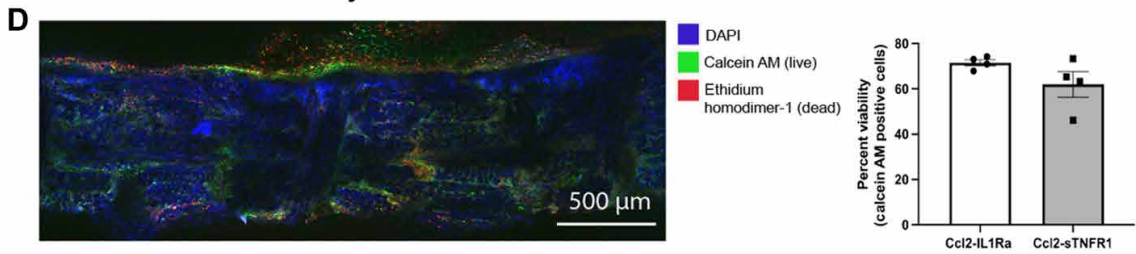

E
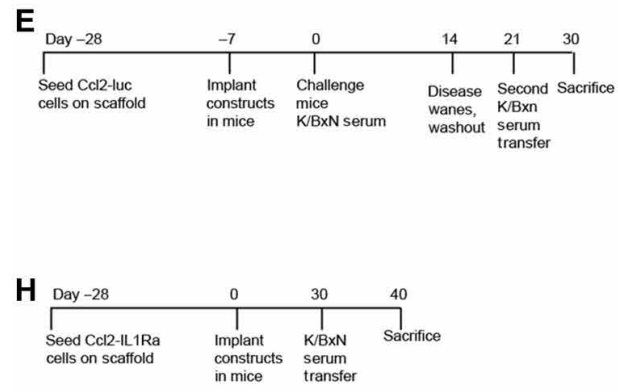

$F$

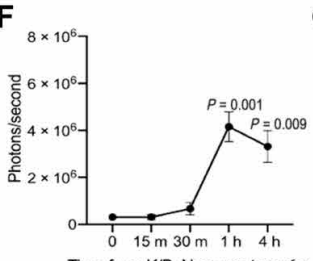

I
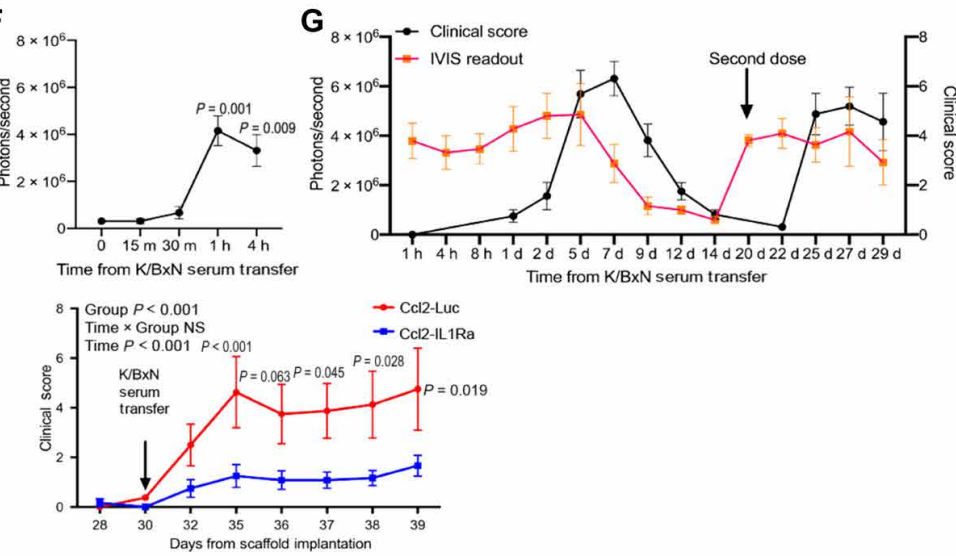

Time from K/BxN serum transfer
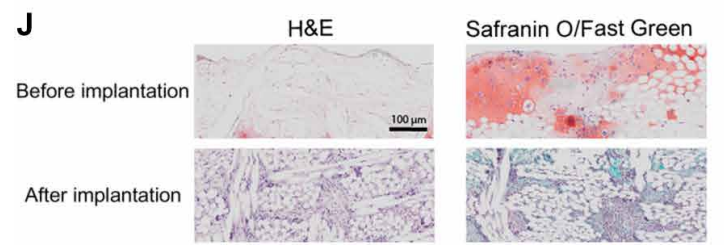

Col I

Col II

Col X
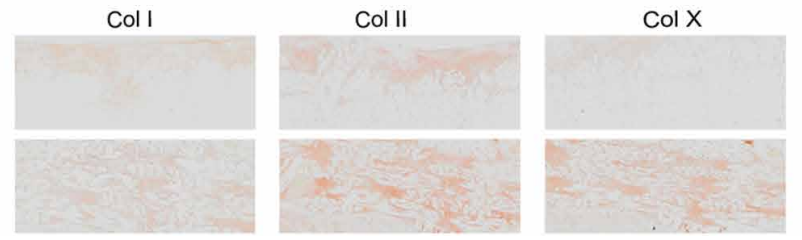

Fig. 4. Bioartificial implants demonstrate translationally relevant longevity, therapeutic efficacy, and retriggering dynamics. (A and B) When implanted in $K / B \times N$ model of RA, Ccl2-Luc implants expressed consistent luciferase and (C) photon flux was similar across subcutaneous implantation on the dorsal aspect or proximal to the knee joint in mice ( $n=3$ per group). (D) Explanted constructs demonstrated high cell viability via confocal microscopy. (E) Ccl2-Luc implants were delivered to mice and (F) challenged to evaluate the on-dynamics where significant increase in photon flux (photons per second) was observed 1 hour after K/BxN STA. (G) Luciferase signal was measured in concert to clinical score over an initial flare, and after a week-long washout period through which minimal signal was detected in concert with attenuated clinical score. The same mice were triggered with a second K/BxN STA flare where a proportional increase in signal was observed. (H and I) An additional group of mice were treated with therapeutic constructs, which laid dormant in mice for 30 days before K/BxN STA, at which time they successfully mitigated disease severity when compared to Ccl2-Luc constructs. (J) H\&E and Safranin O/Fast Green staining of paraffin sections of scaffolds before implantation and after implantation, followed by immunohistochemistry for collagen I, lla1, and X. $n=5$ to 6 per group, two-way ANOVA with Geisser-Greenhouse corrections: different letters indicate $P<0.001$ by Tukey's post hoc test. (B) $n=3$ to 8 , one-way ANOVA; (F) $n=3$ to 5 per group, two-way repeated measures ANOVA with Tukey's post hoc test; (C, G, and I) two-way ANOVA: adjusted $P$ values are shown above each time point for Sidak's post hoc test.

drugs in a self-regulating manner. By engineering iPSCs to form a stable cartilaginous matrix, the implants exhibited extended viability and therapeutic function an in vivo model of RA, with the ability to serve as an autoregulated source for prescribed biologic drugs. The construct could be repeatedly reactivated by inflammatory challenges and showed superiority to standard-of-care treatment with conventional disease-modifying drugs and biologics. Furthermore, these implants can provide direct luminescence readouts of preprogrammed responses, opening up potential applications in real-time diagnostic assessment of the dynamic inflammatory environment.
While protein-based drug delivery, including enzyme-activated systems, have been successful in a number of applications (23-27), such approaches are generally based on repeated injections or on biodegradable materials and thus cannot easily supply continuously biologic molecules for long-term delivery, unlike the rapid dynamic stimulus-response functions that living cells can provide. In previous studies, "designer" or "smart" cell approaches have been used to develop cell therapies for metabolic diseases such as diabetes but have been based on transient transfection or viral gene delivery, resulting in unpredictable gene insertion sites or copy numbers (4). 
A

\begin{tabular}{|c|c|c|c|}
\hline Day -28 & -7 & 0 & 7 \\
\hline $\begin{array}{l}\text { Seed cells } \\
\text { on scaffold }\end{array}$ & $\begin{array}{l}\text { Implant } \\
\text { constructs } \\
\text { in mice }\end{array}$ & $\begin{array}{l}\text { K/BxN } \\
\text { serum } \\
\text { transfer }\end{array}$ & Sacrifice \\
\hline
\end{tabular}

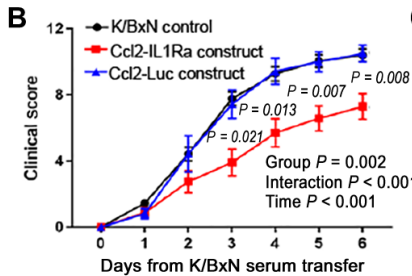

Days from K/BxN serum transfer

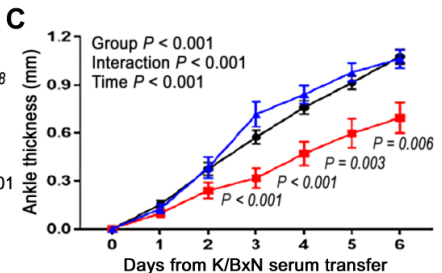

D

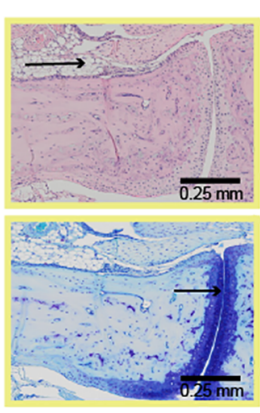

CcI2-IL1Ra construct
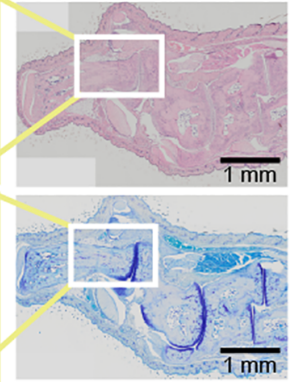

$\mathrm{K} / \mathrm{B} \times \mathrm{N}$ control

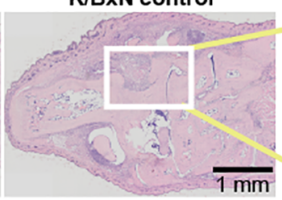

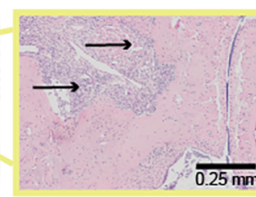

E

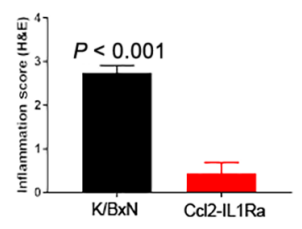

$\mathbf{F}$

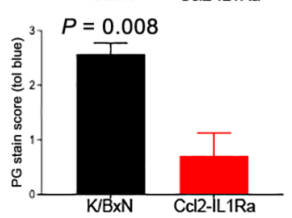

G
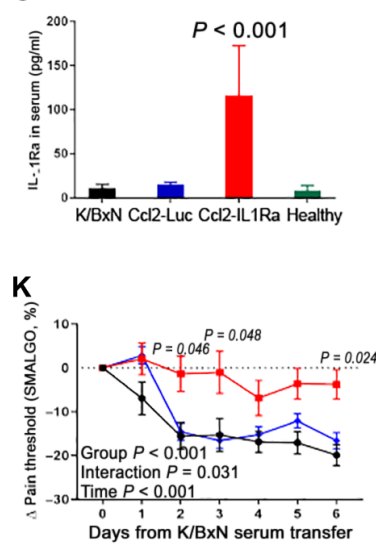

H

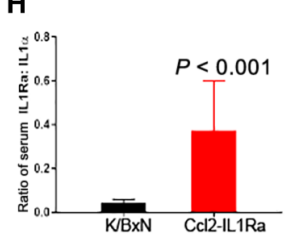

$\mathbf{L}$

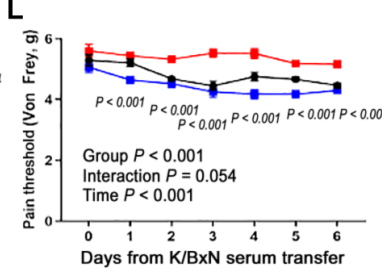

I

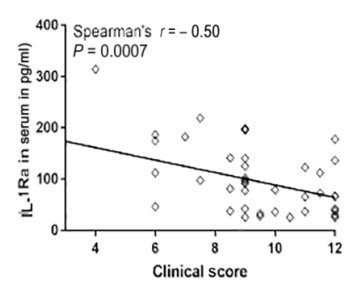

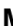

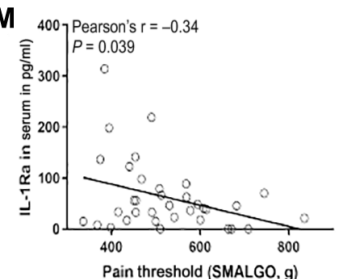

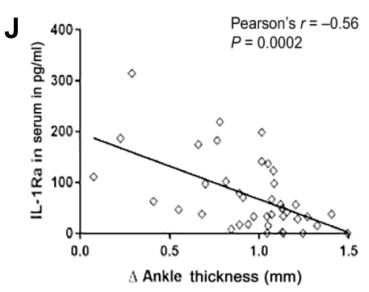

N

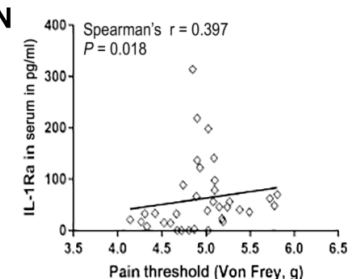

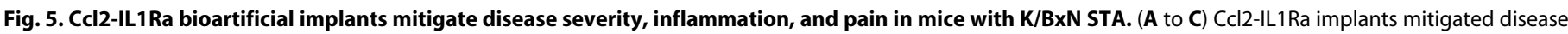

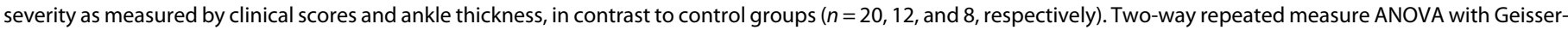

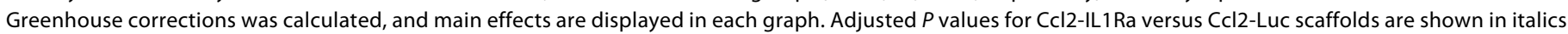

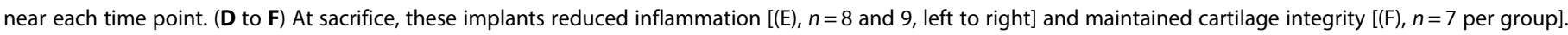

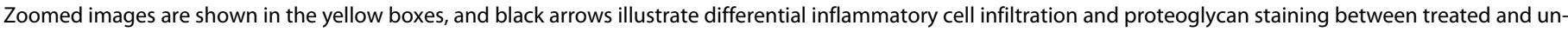

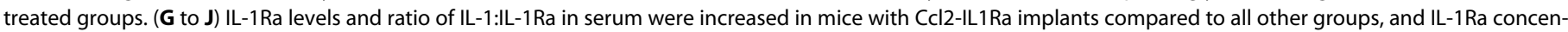

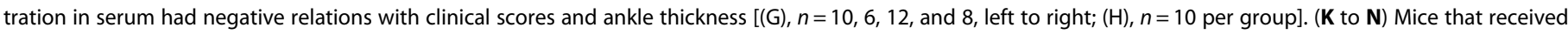

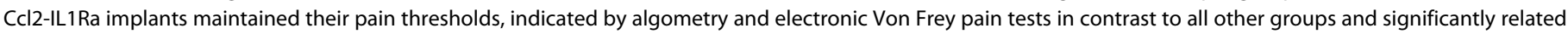

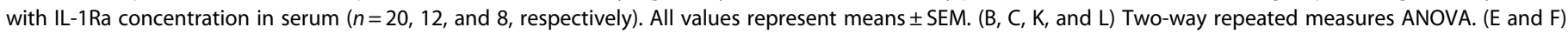
One-sided Mann-Whitney $U$ test. (G) One-way ANOVA. (H) Student's $t$ test.

This study is among the first to demonstrate use of CRISPR-Cas9 gene editing for precise control of the stimulus-response locus in vivo (3), as well as the potential for tunability and genome editing of cytokine receptors for treating model systems of an inflammatory condition $(6,28)$. Recent human studies have illustrated the utility and feasibility of applying CRISPR-Cas9-edited cells in human patients, for example, in a clinical trial of edited autologous $\mathrm{T}$ cells that were found to safely and feasibly treat lung cancer (29). The next frontier of development for applying genome engineering to develop therapeutic cells is to apply tissue engineering in combination with these synthetic biology tools (30) to develop artificial gene circuits that can sense and respond dynamically to disease markers.
The present approach may provide new opportunities for developing safe and effective therapies for a broad range of chronic diseases such as RA (31).

Cell-based therapies may provide the next frontier of medicine by providing systems that can be precisely engineered for highly controlled, long-term drug delivery (32). For example, patients with RA typically require frequent (weekly or biweekly to monthly) systemic dosing of biologic regimens. Thus, a stable implantable drug delivery system could overcome the burden of repeated self-administered injections. This clinical need motivated the investigation of a longer-lasting implant seeded with genome-edited Ccl2-IL1Ra cells to deliver biologic therapeutic over a longer time frame. As the 

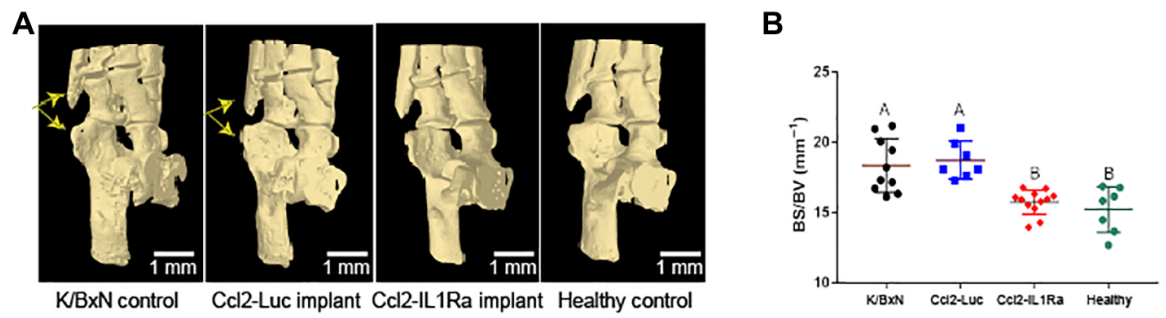

D

E
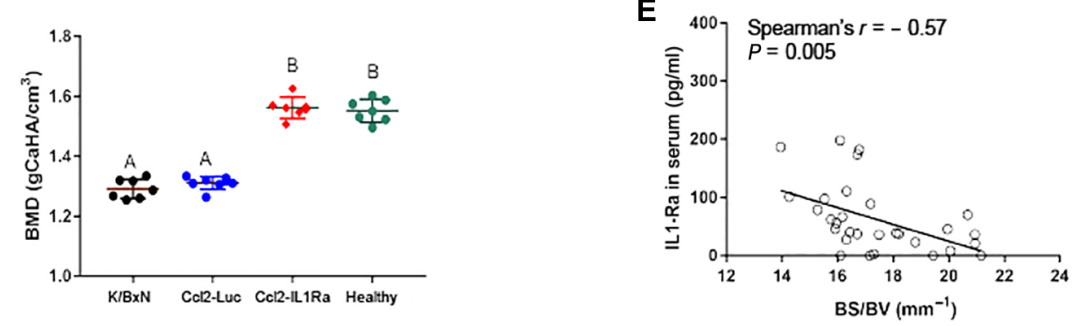

G

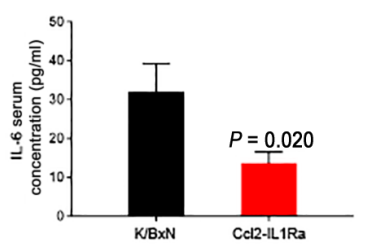

K

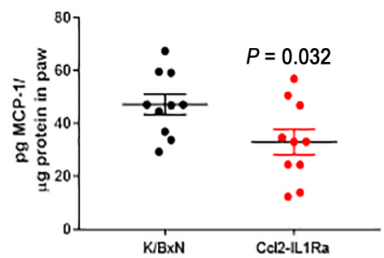

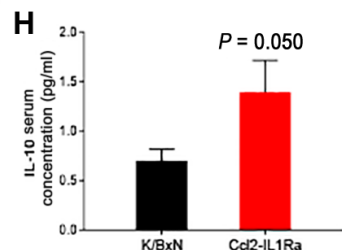

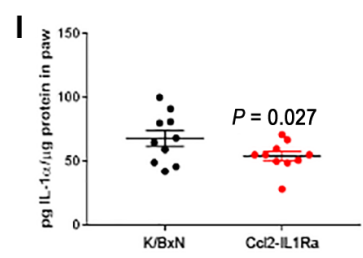

L

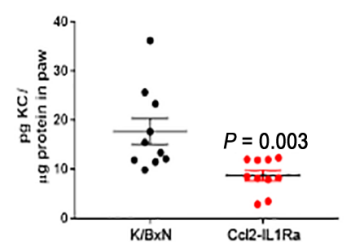

C

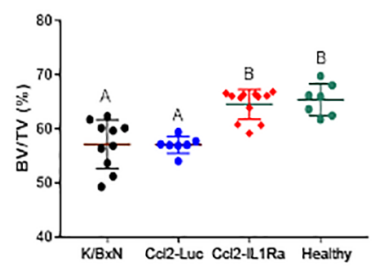

F
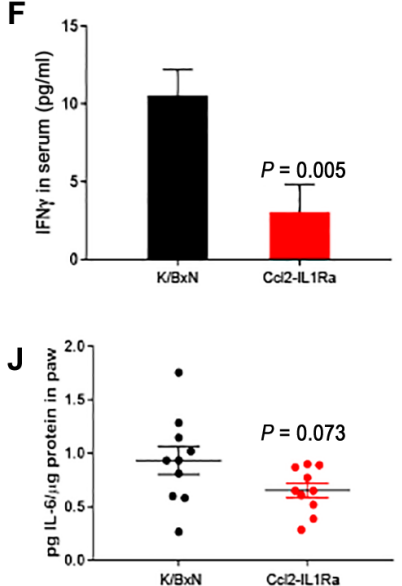

N

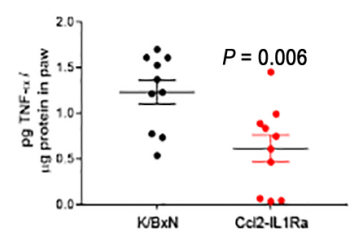

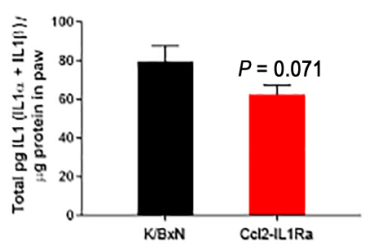

Fig. 6. CCl2-IL1Ra bioartificial implants prevent bone damage and mitigate endogenous inflammation in mice with K/BxN STA. (A to E) Mice treated with CCI2-IL1Ra implants demonstrated reduced bone damage by microCT as quantified by (B) BS/BV, (C) BV/TV, and (D) BMD ( $n=10$, 7, 12, and 7, left to right). (E). Significant relationships were observed between BS/BV and serum levels for IL-1Ra. ( $F$ to $\mathbf{N}$ ) Mice receiving Ccl2-IL1Ra implants also showed less systemic ( $F$ to $H$ ) and local inflammation in lysate from a single hind paw (I to N) as compared to mice with Ccl2-Luc implants or no implants ( $n=10$ per group). All values represent the mean fold change in expression \pm SEM. ( $B$ to $D)$ One-way ANOVA. ( $F$ to N) Student's $t$ test or Mann-Whitney $U$ test. Different letters indicate $P<0.001$ by Tukey's post hoc test.

clinical course of RA is typically punctuated by flares alternating with remissions, an ideal therapeutic strategy should be responsive and effective in blunting the endogenous inflammatory challenges presented during flares. Using 3D woven scaffolds to engineer functional cartilage implants (16), the Ccl2-driven bioengineered cells demonstrated feedback-controlled dynamics in response to fluctuating levels of cytokines commonly implicated in arthritis, suggesting that these bioartificial implants indeed could sense and respond to the in vivo environment perpetuated by $\mathrm{K} / \mathrm{BxN}$ models of inflammatory arthritis. We also observed that the Ccl2-IL1Ra bioartificial implants could be triggered and retriggered to the same magnitude and the circuit was turned "off" when not stimulated, representing advantages that could mitigate adverse events associated with continuous protein administration (2). Given the harsh inflammatory environment in the $\mathrm{K} / \mathrm{BxN} \mathrm{F} 1$ mice, the finding that implants survived and functioned appropriately over 5 weeks in vivo suggests that this approach, with further optimization, could supplant the clinical need of frequent injections of biologic drugs in patients with RA. A limitation of the present study is the absence of longer time points beyond 40 days that will inform the translational potential of this approach. We are currently exploring the delivery of these constructs using other biomaterials that allow for encapsulation of cells at higher densities and long-term survivability, which represent the critical next steps in the development of these Ccl2-IL1Ra and Ccl2-sTNFR1 for anticytokine therapies.

In addition to the significant $\sim 40 \%$ reduction in the inflammatory index conferred by the Ccl2-IL1Ra implants, this approach resulted in complete mitigation of bone erosions. Bone erosions are frequently observed in RA (33) and are associated with poor functional outcomes. However, most biologics currently available in the clinic rarely inhibit both inflammation and bone erosions (33). The ability to mitigate both inflammation and structural damage using this approach suggests that inhibition of IL-1 signaling in an autoregulated manner may provide added therapeutic advantage in the prevention of bone loss and joint destruction in inflammatory arthritis. Recently, a population of $\mathrm{FAP}^{+} \mathrm{THY}^{-}$fibroblasts that produce high levels of matrix metalloproteases (MMPs) have been shown to selectively mediate bony erosions in inflammatory arthritis (34). We demonstrated 


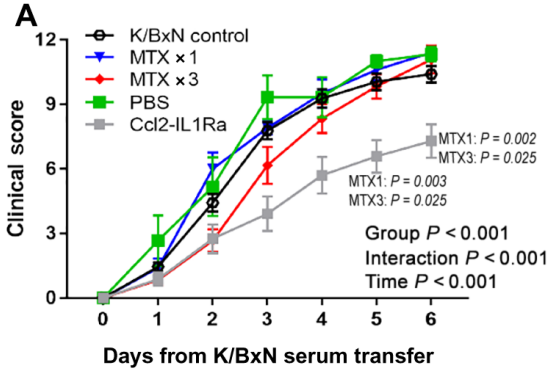

C

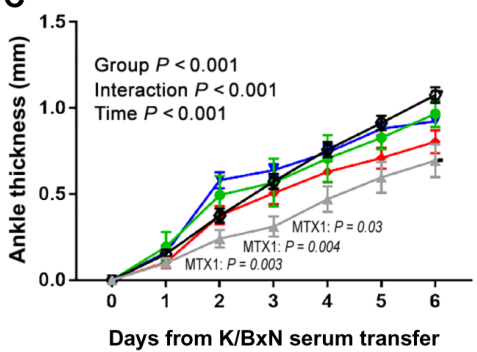

$\begin{aligned} & - \text { CCI2-IL-1Ra } \\ & \neq \text { Tofacitinib }\end{aligned}$

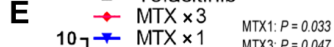

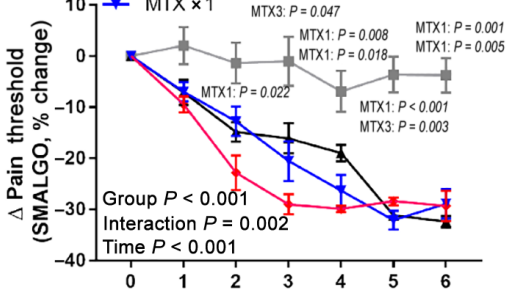

Days from K/BxN serum transfer
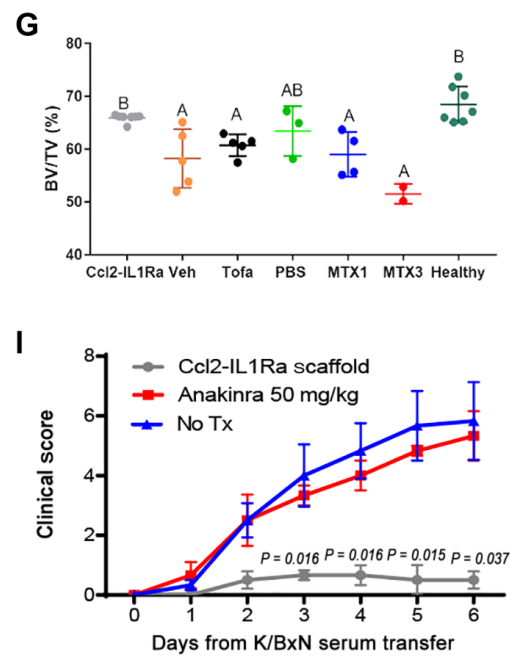

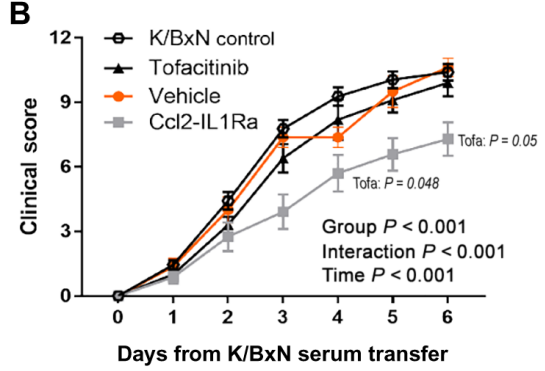

D

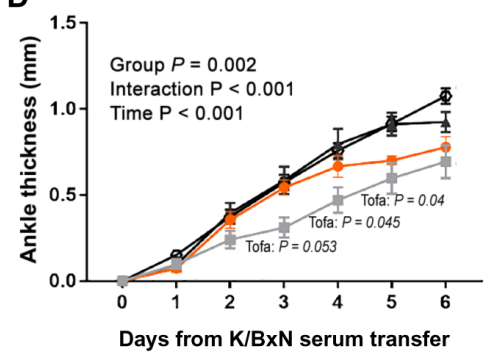

$F$
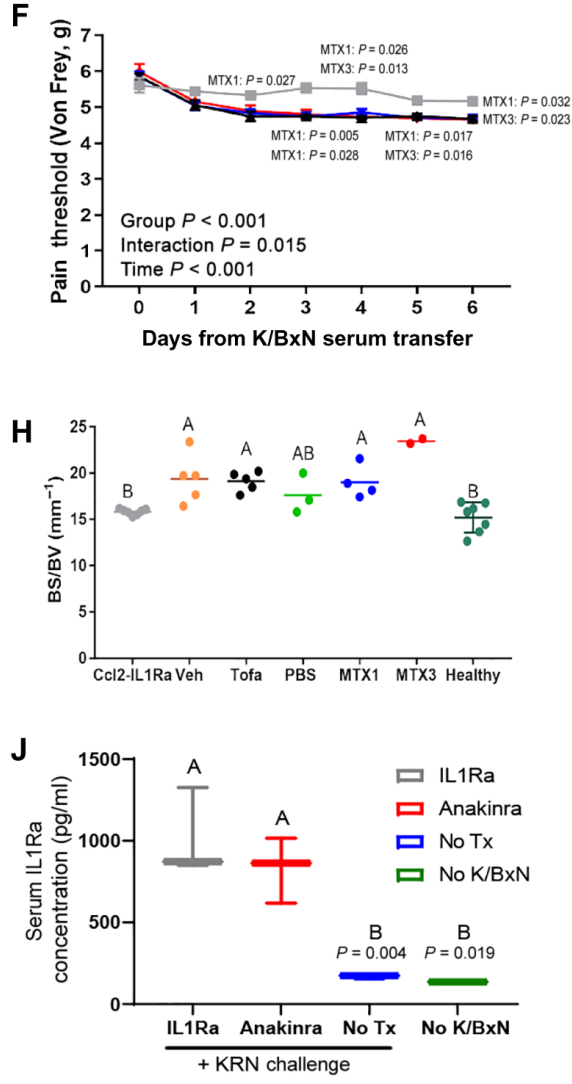

Fig. 7. Ccl2-IL1Ra bioartificial implants demonstrate improved clinical efficacy over standard-of-care treatment. No significant therapeutic benefit was observed with $(\mathbf{A})$ a single intraperitoneal injection of methotrexate $(M T X \times 1)$ on day 2 or three intraperitoneal injections of methotrexate $(M T X \times 3$; on days 2,4 , and 6$)$ or $($ B) tofacitinib twice daily starting on day 2 compared to phosphate-buffered saline (PBS) or vehicle controls. (C and D) Neither benchmark treatment resulted in a significant mitigation of ankle thickness, (D) hyperalgesia, (E) paw withdrawal, (F) Von Frey pain threshold (G) ratio of BS/BV, nor (H) BV/TV to the levels of healthy controls. (I) CCI2-IL1Ra animals demonstrated significantly reduced clinical scores versus anakinra but (J) similar levels of circulating IL-1Ra when measured at day 7 at sacrifice (final dose was administered evening of day 6). (A, C, E, and F) Italicized $P$ values indicate Tukey's test for CCI2-IL1Ra versus MTX1 or MTX3. (B and D) CCI2-IL1Ra versus tofacitinib. (I) CCI2-IL1Ra versus Anakinra at each time point. (J) $P$ values indicate CCl2-IL1Ra versus no treatment and no K/BxN. All values represent means \pm SEM. (A to F) Two-way ANOVA with a Geisser-Greenhouse correction to account for sphericity. ( $\mathrm{G}$ and $\mathrm{H}$ ) One-way ANOVA. Different letters indicate $P<0.05, n=3$ to 8 mice per treatment group. 
in vitro that Ccl2-IL1Ra cells blunted the production of MMP-13 in response to IL-1 $\alpha$ challenge, suggesting that modulation of MMP expression underlies the mechanism by which IL-1 drives the erosive disease in RA.

The bioartificial implants outperformed standard-of-care benchmark comparators evaluated in this context, including methotrexate and tofacitinib. Notably, Ccl2-IL1Ra implants outperformed anakinra, suggesting that cell-based release of therapeutic levels of IL-1Ra on demand in response to endogenous inflammatory signals provides a superior mode of delivery as compared to the standard exogenous delivery of anakinra (e.g., twice daily injections). This finding is consistent with previous studies that suggest that the short half-life and rapid clearance of IL-1Ra following injection may be responsible for the inconsistent clinical efficacy (35).

The present cell-based anticytokine therapy dynamically senses and responds to the environment, so this feature can help overcome the short half-life and rapid clearance, as IL-1 Ra is being synthesized continuously in response to stimulus, rather than being delivered in a single bolus that is rapidly cleared (36). Of note, the level of IL-1Ra in mice implanted with Ccl2-IL1Ra scaffolds and mice receiving anakinra was similarly low at sacrifice, day 7 in mice with scaffolds and $\sim 20$ hours after the last dose of anakinra. However, we reasoned that Ccl2-IL1Ra implants outperformed anakinra because of sustained levels of IL-1Ra released by the Ccl2-IL1Ra cells in direct response to stimulation by endogenous inflammatory mediators in the setting of $\mathrm{K} / \mathrm{BxN}$ serum challenge. The short halflife of anakinra is well known, and the rapid clearance of exogenous anakinra in mice has been documented (36). While the bulk of anakinra is cleared within hours following systemic administration in mice $(36,37)$, IL-1Ra released by a single challenge of IL-1 persists for at least 72 hours (Fig. 3E). In addition, the response of $\mathrm{Ccl} 2$ cells in the K/BxN STA model is sustained for days (Fig. 4G). Thus, the vastly different kinetics of endogenous IL-1Ra released by stimulated Ccl 2 cells and exogenously administered anakinra likely explain the differing efficacy between the two treatments. The in vitro data presented here demonstrate robust and consistent IL-1Ra protein concentrations through 24 hours after a single stimulus. This cell-based approach also includes the time required for protein synthesis of IL-1Ra, as it is transcribed in the cell-based system rather than delivered as a functional protein, which we speculate that this transcription in response to inflammatory stimuli may help overcome the short half-life and rapid clearance of injectable IL-1Ra. However, this hypothesis remains to be directly tested and is the focus of ongoing studies.

Another explanation for the lack of efficacy of clinical DMARDS in this model system may be due to both dosing and altered drug kinetics between IL1Ra in humans and mice may explain the lack of efficacy of standard-of-care drugs in the present studies. Difference in drug kinetics is most evident in methotrexate-treated mice where once a week dosing (standard for RA therapy) shows no effect but every other day dosing three times leads to some mitigation of disease. Tofacitinib, on the other hand, has been shown to be effective in murine collagen-induced arthritis, especially when given continuously via subcutaneous pump administration (38). Lack of efficacy in the K/BxN STA suggests differences in molecular drivers of inflammation between the two models. Because IL-1Ra is the first line of treatment for several additional autoinflammatory diseases (cryopyrin-associated autoinflammatory syndromes, TNF receptorassociated periodic syndrome, familial Mediterranean fever, etc.)
(39), the stable implantable approach may be explored for better control of inflammatory flares in other chronic conditions as well. We recognize that systemic immune suppression is also a commonly reported limitation of biologic drugs for these indications $(40,41)$. Future work will also focus on detailing how the present bioartificial drug delivery system may affect the immune system.

In summary, this cell-based anticytokine therapy represents an advance in the development of therapies for RA. Ccl2-IL1Ra cells seeded on a 3D woven scaffold represent a bioartificial implant system that can overcome many clinical limitations of existing treatment strategies for arthritides. By leveraging the flexibility of iPSCs as a cell source, this approach can be readily expanded to generate therapeutic cells that could be broadly applied and differentiated into any cell type in the future, allowing for additional applications to address a variety of chronic diseases and tissue types where defined biologic targets have been identified. Ongoing work is interrogating other biomaterials and synthetic gene circuits for further tuning of the cellular responses in other chronic inflammatory disease models. Longer-term studies will inform the utility of this approach to other types of arthritis now that proof of concept has been established.

\section{MATERIALS AND METHODS \\ Cell culture and in vitro assays}

Murine iPSCs were engineered to incorporate a synthetic gene circuit to drive the expression of either Il1rn or firefly luciferase $(L u c)$ via the $C c l 2$ locus (3). These iPSCs were predifferentiated in micromass with bone morphogenetic protein 4 (17), seeded onto 3D woven PCL scaffolds $\left(1 \times 10^{6}\right.$ cells per scaffold), and cultured for 21 days in serum-free chondrogenic medium containing Dulbecco's modified Eagle's medium-high glucose, nonessential amino acids, 2-mercaptoethanol, ITS+ premix (BD), penicillin-streptomycin (Gibco), L-ascorbic acid 2-phosphate $(50 \mu \mathrm{g} / \mathrm{ml})$, L-proline $(40 \mu \mathrm{g} / \mathrm{ml})$, transforming growth factor- $\beta 3$ (10 ng/ml; R\&D Systems), and $100 \mathrm{nM}$ dexamethasone $(17,42)$ to make implantable cartilage constructs (16). Gene expression changes in response to inflammatory cytokines $[\mathrm{IL}-1 \alpha(1 \mathrm{ng} / \mathrm{ml})$ or TNF- $\alpha(20 \mathrm{ng} / \mathrm{ml})]$ were measured via quantitative polymerase chain reaction $(17,43)$, and protein levels of secreted murine IL-1Ra were measured via enzyme-linked immunosorbent assay (ELISA) (DuoSet, R\&D Systems).

\section{NanoCT imaging of bioartificial implants}

Implants were fixed overnight in $2.5 \%$ glutaraldehyde and $2 \%$ paraformaldehyde in $0.1 \mathrm{M}$ cacodylate buffer with $2 \mathrm{mM} \mathrm{CaCl}_{2}$. Secondary fixation was performed using $1 \% \mathrm{OsO}_{4}$ in 0.1 cacodylate buffer for 1 hour at room temperature. Excess fixative was washed using deionized water, and the samples were incubated in Lugol's iodine for 72 hours at room temperature to further enhance contrast. Samples were embedded in $2 \%$ agarose and imaged on a Zeiss Xradia Versa $540 \mathrm{x}$-ray microscope using a $0.4 \times$ flat panel detector. Images were reconstructed and presented as shaded volume renderings.

\section{In vivo luciferase assays}

Ccl2-Luc constructs were implanted on the dorsal aspect of F1 K/ $\mathrm{BxN}$ mice, which spontaneously develop chronic arthritis, to allow for longitudinal bioluminescence imaging of luciferase activity of the scaffolds in vivo daily for 1 week, and weekly thereafter over a 5-week period. C57BL/6 mice implanted with Ccl2-Luc scaffold 
constructs were challenged with $\mathrm{K} / \mathrm{BxN}$ serum and imaged daily for 7 days. In vivo bioluminescence imaging was performed on the days indicated on an IVIS Lumina (PerkinElmer, Waltham, MA, USA; Living Image 4.2; 1-min exposure; bin, 8; field of view, $12.5 \mathrm{~cm}$; f/stop, 1; open filter). Hair was removed from the area before imaging. Mice were injected intraperitoneally with D-luciferin $[150 \mathrm{mg} / \mathrm{kg}$ in phosphate-buffered saline (PBS); Gold Biotechnology, St. Louis, MO, USA] and imaged using isoflurane anesthesia (2\% vaporized in $\mathrm{O}_{2}$ ). Total photon flux (photons per second) was measured from fixed regions of interest over the lower back of the mice where the scaffolds were implanted using Living Image 2.6.

\section{Cell viability assays}

Cell survival and apoptosis were assessed using the Live/Dead Cell Viability/Cytotoxicity Kit for mammalian cells (Invitrogen/Molecular Probes, Carlsbad, California, USA). Live cells were labeled with calcein AM, and dead cells were labeled with ethidium homodimer-1 bound to DNA. Labeled constructs were imaged using confocal microscopy (LSM 880, Zeiss, Thornwood, NY, USA) (16). Live/dead expression was quantified in histogram statistics (ZEN blue edition, Zeiss) as the percentage of 488 channel signaling normalized by total signaling from both channels for a flattened $z$ stack collected of the half-construct.

\section{$\mathrm{K} / \mathrm{BxN}$ model of inflammatory arthritis}

Male $\mathrm{K} / \mathrm{BxN}$ transgenic mice and female B6.I- $\mathrm{A}^{\mathrm{g} 7}$ mice were intercrossed to generate F1 that spontaneously developed arthritis beginning at about 4 weeks of age and lasting for $>20$ weeks (15). STA was induced in C57BL/6 male and female mice with 150 to $175 \mu$ of $\mathrm{K} / \mathrm{BxN}$ serum delivered by retro-orbital injection $(14,15,44)$. One week before STA, mice received either subcutaneous Ccl2-IL1Ra scaffold implants on the dorsum $(n=8$ scaffolds, 8 animals, experimental group), Ccl2-Luc scaffolds, or no treatment (control groups, $n=8$ to 13 ). In separate groups of C57BL/ 6 mice, animals were induced with STA and received methotrexate intraperitoneally, tofacitinib by oral gavage, or appropriate controls. Disease activity (clinical score and ankle thickness) and pain sensitivity (algometry and electronic Von Frey) were assessed daily for 1 week. Mice were then sacrificed, and serum and hind paws were collected for analysis. All procedures were approved by the Institutional Animal Care and Use Committee at Washington University in St. Louis.

The clinical score was assessed on a scale of 0 to $3(0$, no swelling or erythema; 1 , slight swelling or erythema; 2 , moderate erythema and swelling in multiple digits or entire paw; and 3, pronounced erythema and swelling of entire paw; maximum total score of 12) (45). The change from baseline in ankle thickness was determined daily by dial calipers, and an average change in the ankle thickness was determined for each mouse from the two hind paw measurements.

\section{Pain and behavioral testing}

Animals were acclimatized to the behavioral testing room before assessment. Algometry was performed using a pressure-based analgesimeter (SMALGO, Bioseb, Vitrolles, France) by applying a progressive force over the ankle joint, and the stimulation was increased until a reaction (shudder or vocalization) from the animal was obtained. The maximum force was then automatically recorded, analyzed, and reported as pressure-pain hyperalgesia.

To determine tactile allodynia, electronic Von Frey paw assays were used. Mice were placed in acrylic cages $(12 \mathrm{~cm}$ by $10 \mathrm{~cm}$ by
$17 \mathrm{~cm}$ high) with a wire grid floor 15 to $30 \mathrm{~min}$ before testing in a quiet room. During this adaptation period, the hind paws were stimulated two to three times. Before hind paw stimulation, the animals were kept quiet, without exploratory movements or defecation, and not resting on their paws. In these experiments, a pressure meter that consisted of a handheld force transducer fitted with a $0.5-\mathrm{mm}^{2}$ polypropylene tip (electronic von Frey anesthesiometer, IITC Inc., Life Science Instruments, Woodland Hills, CA, USA) was used. A tip was applied against the central edge of the animal hind paw, and the intensity of the stimulus was automatically recorded when the paw was withdrawn. The stimulation of the paw was repeated until the animal presented two similar measurements.

\section{Histological analysis}

Paws were harvested on day 7 after serum transfer, fixed in $10 \%$ neutral buffered formalin for 48 hours, and stored in $70 \%$ ethanol, before decalcification in EDTA solution and processing for paraffin embedding. Sections $(5 \mu \mathrm{m})$ were stained with H\&E or toluidine blue. Inflammatory cells infiltrating the synovial lining and the joint cavity were enumerated in 8 to 10 random fields per section using $\mathrm{H} \& \mathrm{E}$ images acquired at $\times 400$ magnification. Proteoglycan loss in the cartilage was scored on toluidine blue-stained sections on a scale from 0 to 3 , ranging from fully stained cartilage (score, 0 ) to fully destained cartilage (score, 3), as previously described (45). Scoring was performed by an observer blinded to the treatment.

\section{Histology and immunohistochemistry of scaffold sections}

Constructs were fixed in $4 \%$ paraformaldehyde, embedded in paraffin, and sectioned at thickness of $5 \mu \mathrm{m}$. For histological assessments, constructs were stained with Safranin O/Fast Green or H\&E according to previous methods (17). Collagen I, II, and X were detected using immunohistochemistry. Paraffin sections of scaffold were rehydrated, washed, and incubated for $20 \mathrm{~min}$ at $37^{\circ} \mathrm{C}$ with proteinase $\mathrm{K}(10 \mu \mathrm{g} / \mathrm{ml}$; BioLabs, catalog no. P8102S) diluted in $10 \mathrm{mM}$ tris- $\mathrm{HCl}(\mathrm{pH} 8.0)$ for antigen retrieval. The slides were then treated with $3 \%$ peroxidase $\mathrm{H}_{2} \mathrm{O}_{2}$ in methanol and blocked with $5 \%$ donkey serum for 1 hour at room temperature. Primary antibodies collagen 1 (Abcam, ab90395), IIa1 (University of Iowa, II-II6B3-s), and X (Sigma-Aldrich, C7974) were diluted in 1\% bovine serum albumin (BSA) to a concentration of $1: 800,1: 1$, and $1: 400$, respectively, and incubated overnight at $4^{\circ} \mathrm{C}$. The secondary antibody, peroxidase-conjugated donkey anti-mouse immunoglobulin $\mathrm{G}$ (Jackson ImmunoResearch, catalog no. 715-035-150), was diluted 1:500 in 1\% BSA and incubated at room temperature for 1 hour. Slides were developed using the HistoMark Orange Peroxidase Substrate Kit (SeraCare, catalog no. 5510-0033), dehydrated, mounted, and imaged. Mouse paws were used as controls for positive staining.

\section{Quantitation of IL-1Ra and proinflammatory mediators}

Levels of IL-1Ra in serum and single hind paws were assessed by ELISA (Quantikine-IL1Ra, R\&D Systems). Levels of paw and serum inflammatory mediators were evaluated using Luminex (Mouse High Sensitivity T cell Discovery Array 18-Plex, Eve Technologies, Calgary, AB, Canada).

\section{MicroCT analysis of bone erosion}

To measure bone morphological changes, intact hind paws were scanned by microCT (SkyScan 1176, Bruker) with a 9- $\mu$ m isotropic 
voxel resolution at $50 \mathrm{kV}, 500 \mu \mathrm{A}, 980$-ms integration time, 3 frame averaging, and $0.5-\mathrm{mm}$ aluminum filter to reduce the effects of beam hardening. Images were reconstructed using NRecon software (with $20 \%$ beam hardening correction and 15-ring artifact correction). Hydroxyapatite calibration phantoms were used to calibrate bone density values (in $\mathrm{g} / \mathrm{cm}^{3}$ ). Parameters reported are as follows: BS/BV, bone fraction (BV/TV), and BMD (in $\mathrm{g} / \mathrm{cm}^{3}$ ).

\section{Benchmark studies}

Methotrexate, the first-line DMARD, and tofacitinib and anakinra, two Food and Drug Administration-approved biologic drugs for $\mathrm{RA}$, were administered in the K/BxN STA to further assess the therapeutic efficacy of Ccl2-IL1Ra constructs compared to standard of care for clinical RA. First, we assessed therapeutic efficacy of tofacitinib ( $5 \mathrm{mg} / \mathrm{kg}$ ), a JAK-signal transducer and activator of transcription inhibitor, given twice daily by oral gavage starting on day 2 . Control mice received a methylcellulose $(0.5 \%$ methylcellulose and $0.023 \%$ Tween 20 in PBS) vehicle control by oral gavage. Tofacitinib citrate (PZ0017, Sigma-Aldrich, St. Louis, MO, USA) was prepared in dimethyl sulfoxide (DMSO) and diluted 1:10 in the vehicle control to a final volume of $10 \%$ DMSO. Note that the standard dose of tofacitinib in the clinic is $5 \mathrm{mg}$ taken orally twice a day. Methotrexate $(2.5 \mathrm{mg} / \mathrm{kg})$ was given as a single intraperitoneal injection on day 2 or as multiple injections on days 2, 4, and 6. Controls received PBS intraperitoneally at the same schedule as methotrexate. Note that the standard dose of methotrexate is $25 \mathrm{mg}$ taken orally once a week. Next, to compare cell-based drug delivery versus conventional constitutive IL-1Ra delivery, anakinra ( $50 \mathrm{mg} / \mathrm{kg}$ ) was administered twice daily via intraperitoneal injection and was compared against $\mathrm{Ccl} 2$ IL1Ra scaffold implants through day 6 . Clinical scores, ankle thickness, and microCT measurements were conducted as described above on day 7 .

\section{Statistical analysis}

Sample size was determined on the basis of a mean clinical score of $10 \pm 2$ for $\mathrm{K} / \mathrm{BxN}$ control animals and six for IL-1Ra-treated animals. On the basis of an alpha of 0.05 and $80 \%$ statistical power $(1-\beta)$ a priori, a sample size of four animals per treatment group was needed to observe this effect. Normality was assessed by group using a Shapiro-Wilk test. Brown-Forsyth test was conducted to evaluate equality of variances. Outcomes were evaluated by two-way Student's $t$ test; one-way Mann-Whitney $U$ test or one-, two-, and three-way repeated measures; or three-way analysis of variance (ANOVA) with a GeisserGreenhouse correction to account for sphericity. Tukey's post hoc test or Sidak correction was used to assess differences between groups, treatments, scaffold types, time, or a combination of those factors. Pearson or Spearman correlations were calculated between serum levels of IL-1Ra and outcomes $(P \leq 0.05)$. Investigators were not blinded to the clinical score or ankle thickness measurements. All other assessments and analyses were performed blinded.

\section{SUPPLEMENTARY MATERIALS}

Supplementary material for this article is available at https://science.org/doi/10.1126/ sciadv.abj 1414

View/request a protocol for this paper from Bio-protocol.

\section{REFERENCES AND NOTES}

1. J. E. Gottenberg, O. Brocq, A. Perdriger, S. Lassoued, J. M. Berthelot, D. Wendling, L. Euller-Ziegler, M. Soubrier, C. Richez, B. Fautrel, A. L. Constantin, X. Mariette, J. Morel,
M. Gilson, G. Cormier, J. H. Salmon, S. Rist, F. Liote, H. Marotte, C. Bonnet, C. Marcelli, J. Sellam, O. Meyer, E. Solau-Gervais, S. Guis, J. M. Ziza, C. Zarnitsky, I. Chary-Valckenaere, O. Vittecoq, A. Saraux, Y. M. Pers, M. Gayraud, G. Bolla, P. Claudepierre, M. Ardizzone, E. Dernis, M. A. Breban, O. Fain, J. C. Balblanc, O. Aberkane, M. Vazel, C. Back, S. Candon, L. Chatenoud, E. Perrodeau, J. Sibilia, P. Ravaud, Non-TNF-targeted biologic vs a second anti-TNF drug to treat rheumatoid arthritis in patients with insufficient response to a first anti-TNF drug: A randomized clinical trial. JAMA 316, 1172-1180 (2016).

2. S. Tarp, D. E. Furst, M. Boers, G. Luta, H. Bliddal, U. Tarp, K. H. Asmussen, B. Brock, A. Dossing, T. S. Jorgensen, S. Thirstrup, R. Christensen, Risk of serious adverse effects of biological and targeted drugs in patients with rheumatoid arthritis: A systematic review meta-analysis. Rheumatology 56, 417-425 (2017).

3. J. M. Brunger, A. Zutshi, V. P. Willard, C. A. Gersbach, F. Guilak, Genome engineering of stem cells for autonomously regulated, closed-loop delivery of biologic drugs. Stem Cell Reports 8, 1202-1213 (2017).

4. H. Ye, M. Xie, S. Xue, G. Charpin-El Hamri, J. Yin, H. Zulewski, M. Fussenegger, Selfadjusting synthetic gene circuit for correcting insulin resistance. Nat. Biomed. Eng. 1, 0005 (2017).

5. N. Joshi, J. Yan, S. Levy, S. Bhagchandani, K. V. Slaughter, N. E. Sherman, J. Amirault, Y. Wang, L. Riegel, X. He, T. S. Rui, M. Valic, P. K. Vemula, O. R. Miranda, O. Levy, E. M. Gravallese, A. O. Aliprantis, J. Ermann, J. M. Karp, Towards an arthritis flareresponsive drug delivery system. Nat. Commun. 9, 1275 (2018).

6. J. M. Brunger, A. Zutshi, V.P. Willard, C. A. Gersbach, F. Guilak, CRISPR/Cas9 editing of murine induced pluripotent stem cells for engineering inflammation-resistant tissues. Arthritis Rheumatol. 69, 1111-1121 (2017).

7. J. A. Doudna, E. Charpentier, The new frontier of genome engineering with CRISPR-Cas9. Science 346, 1258096 (2014)

8. T. Gaj, C. A. Gersbach, C. F. Barbas III, ZFN, TALEN, and CRISPR/Cas-based methods for genome engineering. Trends Biotechnol. 31, 397-405 (2013).

9. S. Hao, D. Baltimore, The stability of mRNA influences the temporal order of the induction of genes encoding inflammatory molecules. Nat. Immunol. 10, 281-288 (2009).

10. A. D. Christensen, C. Haase, A. D. Cook, J. A. Hamilton, K/BxN serum-transfer arthritis as a model for human inflammatory arthritis. Front. Immunol. 7, 213 (2016).

11. B. Bresnihan, Treatment of rheumatoid arthritis with interleukin 1 receptor antagonist. Ann. Rheum. Dis. 58(Suppl 1), 196-198 (1999).

12. S. B. Abramson, A. Amin, Blocking the effects of IL-1 in rheumatoid arthritis protects bone and cartilage. Rheumatology 41, 972-980 (2002).

13. M. B. Murphy, K. Moncivais, A. I. Caplan, Mesenchymal stem cells: Environmentally responsive therapeutics for regenerative medicine. Exp. Mol. Med. 45, e54 (2013).

14. V. Kouskoff, A. S. Korganow, V. Duchatelle, C. Degott, C. Benoist, D. Mathis, Organ-specific disease provoked by systemic autoimmunity. Cell 87, 811-822 (1996).

15. D. Kyburz, M. Corr, The KRN mouse model of inflammatory arthritis. Springer Semin. Immunopathol. 25, 79-90 (2003).

16. F. T. Moutos, K. A. Glass, S. A. Compton, A. K. Ross, C. A. Gersbach, F. Guilak, B. T. Estes, Anatomically shaped tissue-engineered cartilage with tunable and inducible anticytokine delivery for biological joint resurfacing. Proc. Natl. Acad. Sci. U.S.A. 113, E4513-E4522 (2016).

17. B. O. Diekman, N. Christoforou, V. P. Willard, H. Sun, J. Sanchez-Adams, K. W. Leong F. Guilak, Cartilage tissue engineering using differentiated and purified induced pluripotent stem cells. Proc. Natl. Acad. Sci. U.S.A. 109, 19172-19177 (2012).

18. A. Atala, L. G. Cima, W. Kim, K. T. Paige, J. P. Vacanti, A. B. Retik, C. A. Vacanti, Injectable alginate seeded with chondrocytes as a potential treatment for vesicoureteral reflux. J. Urol. 150, 745-747 (1993).

19. E. A. Makris, D. J. Responte, N. K. Paschos, J. C. Hu, K. A. Athanasiou, Developing functional musculoskeletal tissues through hypoxia and lysyl oxidase-induced collagen cross-linking. Proc. Natl. Acad. Sci. U.S.A. 111, E4832-E4841 (2014).

20. R. L. Mauck, C. C. Wang, E. S. Oswald, G. A. Ateshian, C. T. Hung, The role of cell seeding density and nutrient supply for articular cartilage tissue engineering with deformational loading. Osteoarthr. Cartil. 11, 879-890 (2003).

21. G. Vunjak-Novakovic, I. Martin, B. Obradovic, S. Treppo, A. J. Grodzinsky, R. Langer, L. E. Freed, Bioreactor cultivation conditions modulate the composition and mechanical properties of tissue-engineered cartilage. J. Orthop. Res. 17, 130-138 (1999).

22. E. B. Lee, R. Fleischmann, S. Hall, B. Wilkinson, J. D. Bradley, D. Gruben, T. Koncz, S. Krishnaswami, G. V. Wallenstein, C. Zang, S. H. Zwillich, R. F. van Vollenhoven, O. S. Investigators, Tofacitinib versus methotrexate in rheumatoid arthritis. N. Engl. J. Med. 370, 2377-2386 (2014).

23. A. A. Aimetti, M. W. Tibbitt, K. S. Anseth, Human neutrophil elastase responsive delivery from poly(ethylene glycol) hydrogels. Biomacromolecules 10, 1484-1489 (2009).

24. Y. Brudno, D. J. Mooney, On-demand drug delivery from local depots. J. Control. Release 219, 8-17 (2015)

25. M. Ehrbar, S. C. Rizzi, R. G. Schoenmakers, B. S. Miguel, J. A. Hubbell, F. E. Weber, M. P. Lutolf, Biomolecular hydrogels formed and degraded via site-specific enzymatic reactions. Biomacromolecules 8, 3000-3007 (2007). 
26. R. Langer, Implantable controlled release systems. Pharmacol. Ther. 21, 35-51 (1983).

27. R. J. Nims, L. Pferdehirt, N. B. Ho, A. Savadipour, J. Lorentz, S. Sohi, J. Kassab, A. K. Ross, C. J. O'Conor, W. B. Liedtke, B. Zhang, A. L. McNulty, F. Guilak, A synthetic mechanogenetic gene circuit for autonomous drug delivery in engineered tissues. Sci. Adv. 7, eabd9858 (2021).

28. N. Farhang, J. M. Brunger, J. D. Stover, P. I. Thakore, B. Lawrence, F. Guilak, C. A. Gersbach, L. A. Setton, R. D. Bowles, CRISPR-based epigenome editing of cytokine receptors for the promotion of cell survival and tissue deposition in inflammatory environments. Tissue Eng. Part A 23, 738-749 (2017).

29. Y. Lu, J. Xue, T. Deng, X. Zhou, K. Yu, L. Deng, M. Huang, X. Yi, M. Liang, Y. Wang, H. Shen, R. Tong, W. Wang, L. Li, J. Song, J. Li, X. Su, Z. Ding, Y. Gong, J. Zhu, Y. Wang, B. Zou, Y. Zhang, Y. Li, L. Zhou, Y. Liu, M. Yu, Y. Wang, X. Zhang, L. Yin, X. Xia, Y. Zeng, Q. Zhou, B. Ying, C. Chen, Y. Wei, W. Li, T. Mok, Safety and feasibility of CRISPR-edited T cells in patients with refractory non-small-cell lung cancer. Nat. Med. 26, 732-740 (2020).

30. T. L. Deans, D. W. Grainger, M. Fussenegger, Synthetic biology: Innovative approaches for pharmaceutics and drug delivery. Adv. Drug Deliv. Rev. 105, 1-2 (2016).

31. Z. Chen, A. Bozec, A. Ramming, G. Schett, Anti-inflammatory and immune-regulatory cytokines in rheumatoid arthritis. Nat. Rev. Rheumatol. 15, 9-17 (2019).

32. M. A. Fischbach, J. A. Bluestone, W. A. Lim, Cell-based therapeutics: The next pillar of medicine. Sci. Transl. Med. 5, 179ps7 (2013).

33. G. Schett, E. Gravallese, Bone erosion in rheumatoid arthritis: Mechanisms, diagnosis and treatment. Nat. Rev. Rheumatol. 8, 656-664 (2012).

34. A. P. Croft, J. Campos, K. Jansen, J. D. Turner, J. Marshall, M. Attar, L. Savary, C. Wehmeyer, A. J. Naylor, S. Kemble, J. Begum, K. Durholz, H. Perlman, F. Barone, H. M. McGettrick, D. T. Fearon, K. Wei, S. Raychaudhuri, I. Korsunsky, M. B. Brenner, M. Coles, S. N. Sansom, A. Filer, C. D. Buckley, Distinct fibroblast subsets drive inflammation and damage in arthritis. Nature 570, 246-251 (2019).

35. C. A. Dinarello, A. Simon, J. W. van der Meer, Treating inflammation by blocking interleukin-1 in a broad spectrum of diseases. Nat. Rev. Drug Discov. 11, 633-652 (2012).

36. J. Petrasek, S. Bala, T. Csak, D. Lippai, K. Kodys, V. Menashy, M. Barrieau, S. Y. Min, E. A. Kurt-Jones, G. Szabo, IL-1 receptor antagonist ameliorates inflammasomedependent alcoholic steatohepatitis in mice. J. Clin. Invest. 122, 3476-3489 (2012).

37. N. E. Powers, B. Swartzwelter, C. Marchetti, D. M. de Graaf, A. Lerchner, M. Schlapschy, R. Datar, U. Binder, C. K. Edwards III, A. Skerra, C. A. Dinarello, PASylation of IL-1 receptor antagonist (IL-1Ra) retains IL-1 blockade and extends its duration in mouse urate crystal-induced peritonitis. J. Biol. Chem. 295, 868-882 (2020).

38. M. E. Dowty, M. I. Jesson, S. Ghosh, J. Lee, D. M. Meyer, S. Krishnaswami, N. Kishore, Preclinical to clinical translation of tofacitinib, a Janus kinase inhibitor, in rheumatoid arthritis. J. Pharmacol. Exp. Ther. 348, 165-173 (2014).

39. A. A. Jesus, R. Goldbach-Mansky, IL-1 blockade in autoinflammatory syndromes. Annu. Rev. Med. 65, 223-244 (2014).

40. M. Klimak, R. J. Nims, L. Pferdehirt, K. H. Collins, N. S. Harasymowicz, S. J. Oswald, L. A. Setton, F. Guilak, Immunoengineering the next generation of arthritis therapies. Acto Biomater. S1742-7061(21)00212-9 (2021).
41. H. Malcova, Z. Strizova, T. Milota, I. Striz, A. Sediva, D. Cebecauerova, R. Horvath, IL-1 inhibitors in the treatment of monogenic periodic fever syndromes: From the past to the future perspectives. Front. Immunol. 11, 619257 (2020).

42. B. Johnstone, T. M. Hering, A. I. Caplan, V. M. Goldberg, J. U. Yoo, In vitro chondrogenesis of bone marrow-derived mesenchymal progenitor cells. Exp. Cell Res. 238, 265-272 (1998).

43. L. Pferdehirt, A. K. Ross, J. M. Brunger, F. Guilak, A synthetic gene circuit for self-regulating delivery of biologic drugs in engineered tissues. Tissue Eng. Part A 25, 809-820 (2019).

44. A. S. Korganow, H. Ji, S. Mangialaio, V. Duchatelle, R. Pelanda, T. Martin, C. Degott, H. Kikutani, K. Rajewsky, J. L. Pasquali, C. Benoist, D. Mathis, From systemic T cell self-reactivity to organ-specific autoimmune disease via immunoglobulins. Immunity 10, 451-461 (1999).

45. H. F. Zhou, H. W. Chan, S. A. Wickline, G. M. Lanza, C. T. Pham, $\alpha_{v} \beta_{3}$-targeted nanotherapy suppresses inflammatory arthritis in mice. FASEB J. 23, 2978-2985 (2009).

Acknowledgments: We thank C. Gersbach for important discussions in the early stages of this work and S. Oswald for providing technical writing and analytical support for the manuscript. Funding: This study was supported, in part, by the Shriners Hospitals for Children; the Nancy Taylor Foundation for Chronic Diseases; the Arthritis Foundation; NIH grants AG15768, AG46927, AR072999, AR076820, OD10707, OD021694, DK108742, AR073752, AR074992 AR067491, AR075899, P50 CA094056 (Molecular Imaging Center), OD021694 and NCI P30 CA091842 (Siteman Cancer Center Small Animal Cancer Imaging shared resource); and the Philip and Sima Needleman Fellowship from the Washington University Center of Regenerative Medicine. Author contributions: Y.-R.C., K.H.C., C.T.N.P., and F.G. conceived the project. Y.-R.C., K.H.C., C.-L.W., J.M.B., C.T.N.P., and F.G. designed the experiments. Y.-R.C., K.H.C., L.P., A.K.R., F.T.M., and C.-L.W. conducted in vitro studies. In vivo experiments and data analyses were performed by Y.-R.C., K.H.C., L.E.S., L.P., A.K.R., N.S.H., and C.-L.W. Y.-R.C., K.H.C., and F.G. wrote the manuscript. All the authors read, edited, and approved the final manuscript. Competing interests: F.G. and F.T.M. are employees and shareholders of Cytex Therapeutics Inc. F.G. and J.M.B. are inventors on a patent related to this work filed by Duke University (no. 20180201951, filed 20 November 2015, published 19 July 2018). The authors declare that they have no other competing interests. Data and materials availability: All data needed to evaluate the conclusions in the paper are present in the paper and/or the Supplementary Materials. The cell lines used in this study can be provided by Washington University pending scientific review and a completed material transfer agreement. Requests for the cells lines should be submitted to guilak@wustl.edu.

Submitted 22 April 2021

Accepted 19 July 202

Published 1 September 2021

$10.1126 /$ sciadv.abj1414

Citation: Y.-R. Choi, K. H. Collins, L. E. Springer, L. Pferdehirt, A. K. Ross, C.-L. Wu, F. T. Moutos, N. S. Harasymowicz, J. M. Brunger, C. T. N. Pham, F. Guilak, A genome-engineered bioartificial implant for autoregulated anticytokine drug delivery. Sci. Adv. 7, eabj1414 (2021). 